\title{
Characterization of Zinc Oxide Nanolaminate Films
}

\author{
B. J. Oleson, L. M. Bilke, J. S. Krueger, S. T. King \\ Department of Physics, University of Wisconsin - La Crosse
}

\begin{abstract}
This project sought to increases the understanding of fundamental properties of the transparent conducting oxide materials needed to improve solar energy device efficiency. Specifically, this study focused on the development of $\mathrm{Cu}: \mathrm{ZnO}$ nanolamintate materials where $\mathrm{Cu}$ and $\mathrm{ZnO}$ form alternating layers on the nanomerter scale. Initial results show no intermixing of materials, and suggest that these nanolaminates may be employed as infrared mirrors.
\end{abstract}

\section{Introduction}

With the earth's current energy supplies dwindling at an accelerating rate, it seems obvious that renewable energy is necessary for the future of mankind. Being abundant and relatively low cost, solar energy may be the most feasible route for clean and renewable energy. It is estimated that the earth's population will double within the next 50 years and create a 1.5-3-fold increase in energy demand [1]. With the population growing, space colonization is a topic receiving more focus. Seeing as solar panels are already used to power crafts in space including the international space station, it is reasonable to believe any colonization efforts will make use of solar energy. Also, it has been proposed that large photovoltaic grids be built in space to harness the energy and transmit it to earth in the form of microwaves. To lower the cost of such a project, thin-film solar cells must be employed.

Although solar cells can be found all around the world, the majority of the suns energy goes unharnessed. The amount of solar energy reaching the surface of the planet is so vast that in one year it is about twice as much as will ever be obtained from all of the Earth's non-renewable resources [2]. Before solar panels can be employed on a large scale on earth or in space, significant challenges in material physics and chemistry must be overcome. Thin film solar cells require both an absorbing medium as well as a transparent medium with good electrical conductivity to extract electrons. Currently, indium doped tin oxide (ITO) makes up the transparent conducting oxide (TCO) layer. One setback of thin films is that indium is about as rare and expensive as silver. High cost of solar panels make them more popular in wealthier nations such as Europe and North America, which are countries poorly suited for large scale photovoltaic deployment. This project works on replacing indium with a cheaper material so that the cost of these tools can be drastically reduced and the potential of solar panels can better be realized.

Zinc oxide is a safe and inexpensive material that shows great promise for replacing the ITO used in the TCO layer of modern photovoltaic devices [3]. Although $\mathrm{ZnO}$ has a large and direct band gap desired for photovoltaic devices, it does not display a sufficiently low resistance for solar cell applications. This project aims to understand and overcome the difficulties $\mathrm{ZnO}$ possess and observe the effect annealing has on the material. Specifically it focuses on $\mathrm{ZnO} / \mathrm{Cu}$ nanolaminate films. 


\section{Experimental Results}

The films were grown on Si and micro-glass substrates with via a reactive DC sputter deposition apparatus outfitted with two independently controlled sputter heads. Due to its flexibilities in the control of composition and microstructure, magnetron sputtering is widely employed [4]. One sputter head consisted of an aluminum doped zinc target (Zn:Al/96\%:4\%) and the other contained a copper metal target $(\mathrm{Cu} / 99.99 \%)$. $\mathrm{Al}$ is added to the $\mathrm{Zn}$ target to add free electrons and ultimately increase its conductivity. Two substrates were used to complement each other and overcome physical properties each substrate possesses that restrain future testing of the thin films. Silicon's high melting point of $1414^{\circ} \mathrm{C}$ was utilized to study the effects of annealing and the micro-glass grown samples were used to study transparency. Before the substrates were used in the sputter apparatus they were cut into pieces approximately $1 \mathrm{~cm} \times 2.5 \mathrm{~cm}$, ultrasonic cleaned in acetone, ultrasonic cleaned in alcohol, rinsed in alcohol, and dried. The sputter system has a base pressure of $10^{-5}$ torr which is raised to $10^{-3}$ torr during deposition. All deposition occurred at an emission current of $20 \mathrm{~mA}$ with a 8:1 Argon: $\mathrm{O}_{2}$ ratio of sputter gasses. Argon was used as a sputter gas because it is inert and will not react with the materials being deposited, but its relatively large mass results in an efficient sputter yield. The preferential amount of oxygen was allowed into the chamber to react with $\mathrm{Zn}$ and create $\mathrm{ZnO}$.

Before $\mathrm{ZnO} / \mathrm{Cu}$ nanolaminate films could be grown and studied, a thick sample of pure $\mathrm{ZnO}$ was grown and analyzed with X-ray diffraction (XRD) to confirm its content and purity. As seen in Figure 1 the diffraction pattern shows only $\mathrm{ZnO}$ present with no impurities, including zinc metal.

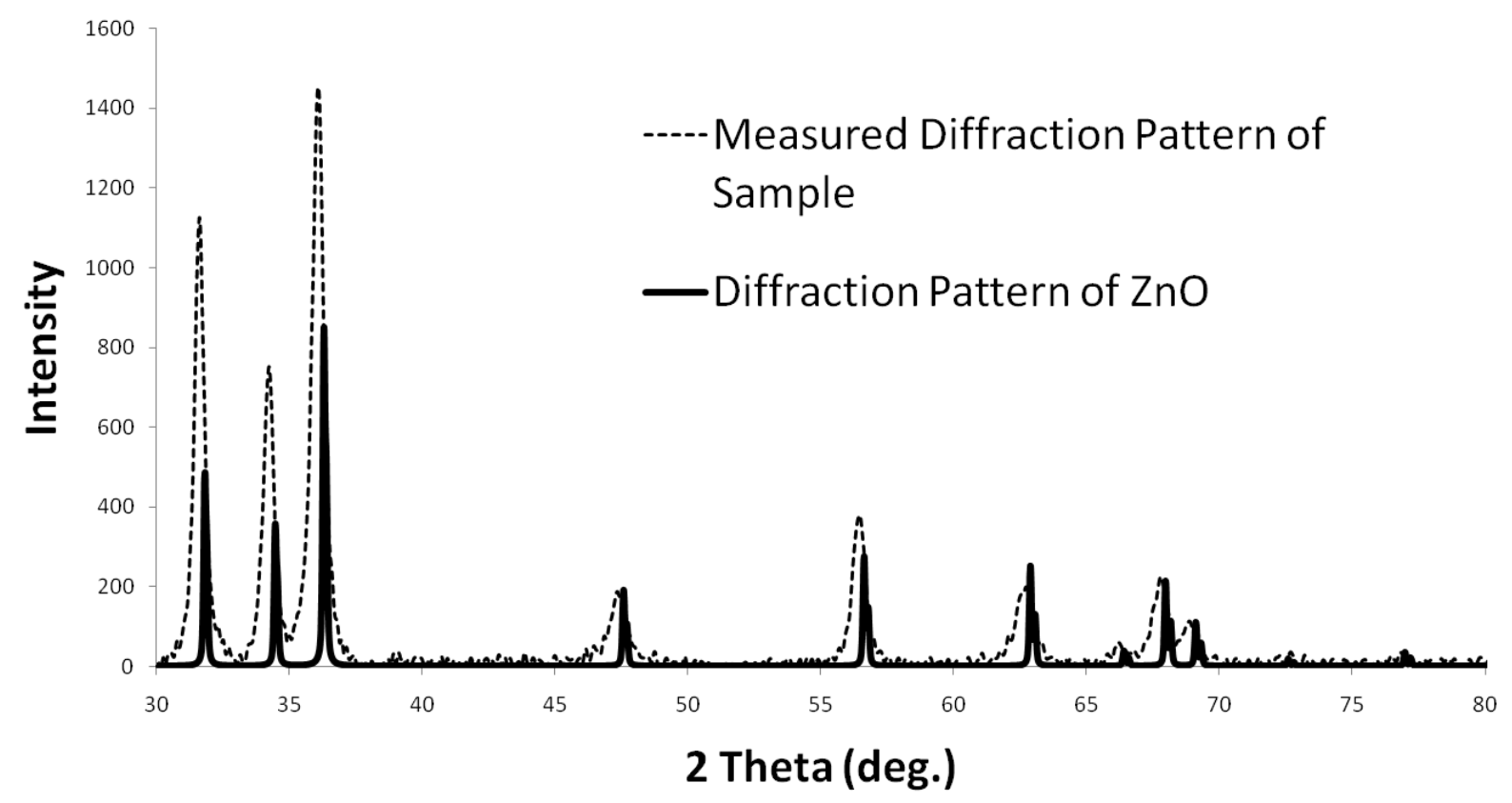

Figure 1. Comparison of XRD patterns, measured and known, reveal a successful growth of $\mathrm{ZnO}$. 
After $\mathrm{ZnO}$ was successfully grown $\mathrm{Cu}$ could be incorporated. A six layer (3 $\mathrm{CU}, 3 \mathrm{ZnO})$ nanolaminate thin film was created by first depositing a $\mathrm{Cu}$ layer directly onto the substrate. A layer of $\mathrm{ZnO}$ was then put on top of the $\mathrm{Cu}$ and the process repeated until the sixth layer was complete. To study the effects the $\mathrm{Cu}$ thickness has on the $\mathrm{ZnO}$ based film, the $\mathrm{ZnO}$ layers in all of the samples were held at $40 \mathrm{~nm}$ each, or a total of $120 \mathrm{~nm}$ per thin film. The Cu layers contained the same thicknesses within each sample but $\mathrm{Cu}$ thicknesses were varied between. XRD performed on the fabricated films confirm the presence of both $\mathrm{Cu}$ and $\mathrm{ZnO}$ with no evidence of the formation of impurity species (Fig. 2).

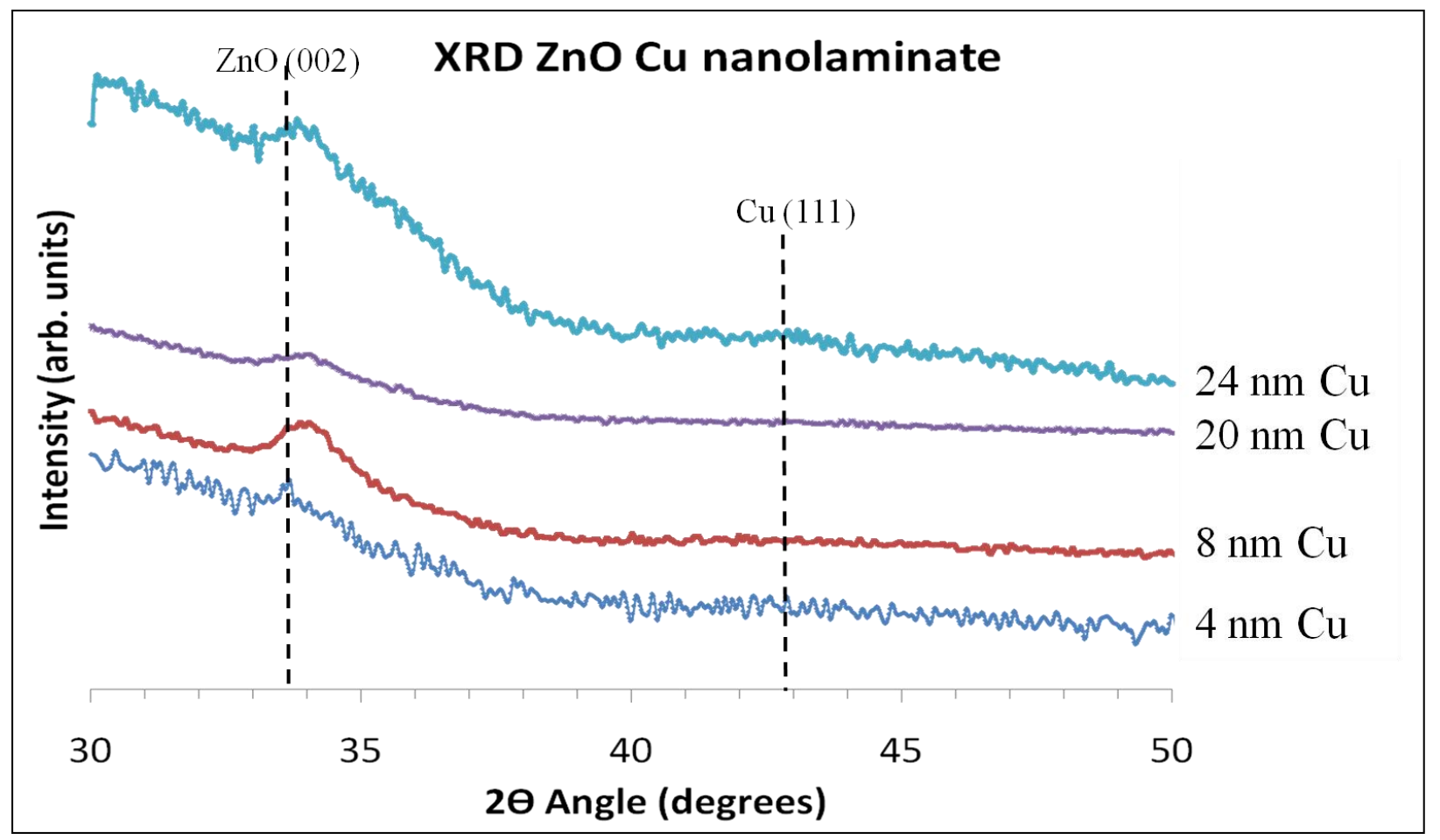

Figure 2. XRD of nanolaminate films shows definite presence of $\mathrm{ZnO}$ and possibly $\mathrm{Cu}$ in the thicker grown samples.

The plot in Figure 2 shows $\mathrm{ZnO}$ is present but it is unclear whether $\mathrm{Cu}$ exists within the film. It appears that when the $\mathrm{Cu}$ layer is thickest $(24 \mathrm{~nm})$ a characteristic $\mathrm{Cu}$ diffraction peak can be made out. X-ray fluorescence was used as a complementary technique to confirm elemental make-up of the film. Although $\mathrm{Cu}$ presence cannot be confirmed in the thinner films in Figure 2, Figure 3 clearly shows both $\mathrm{ZnO}$ and $\mathrm{Cu}$ exist within the film. 


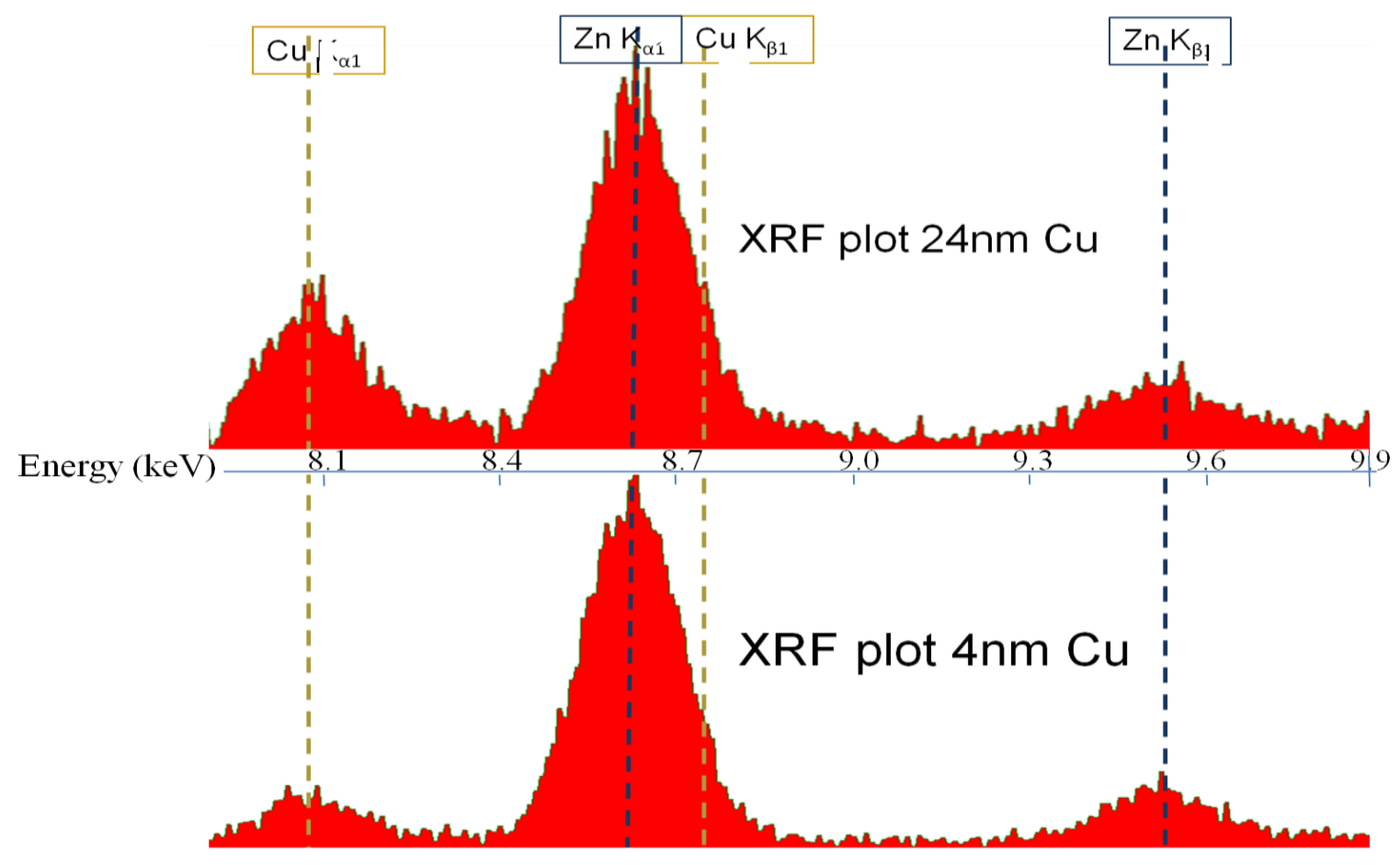

Figure 3. XRF plots of samples with thin and thick layers of $\mathrm{Cu}$ are compared.

An important characteristic of a TCO is its transparency. In this study UV-Vis spectroscopy was used to study the transparency for different thicknesses of $\mathrm{Cu}$ in the films. Figure 4 shows the amount of light transmitted, within the visible spectrum, through these films. These measurements indicate that the films containing $8 \mathrm{~nm}$ and $4 \mathrm{~nm}$ of $\mathrm{Cu}$ exhibit excellent transparency which is more than adequate for TCO applications.



Figure 4. $\mathrm{ZnO} / \mathrm{Cu}$ nanolaminate UV-Vis Spectroscopy comparing transmission for different thicknesses of Cu. 
The next phase of this study required an atomic force microscope (AFM) to study the surface of films and how the surface morphology changes via annealing. Before the nanolaminate films were studied, surface changes of $\mathrm{ZnO}$ due to annealing were first explored. The films were heated to $450^{\circ} \mathrm{C}$ in a tube furnace and evaluated every 30 minutes. The surface didn't change significantly from 0-60 minutes annealed. However, from 60-90 minutes drastic changes were noted as seen in Figure 5.
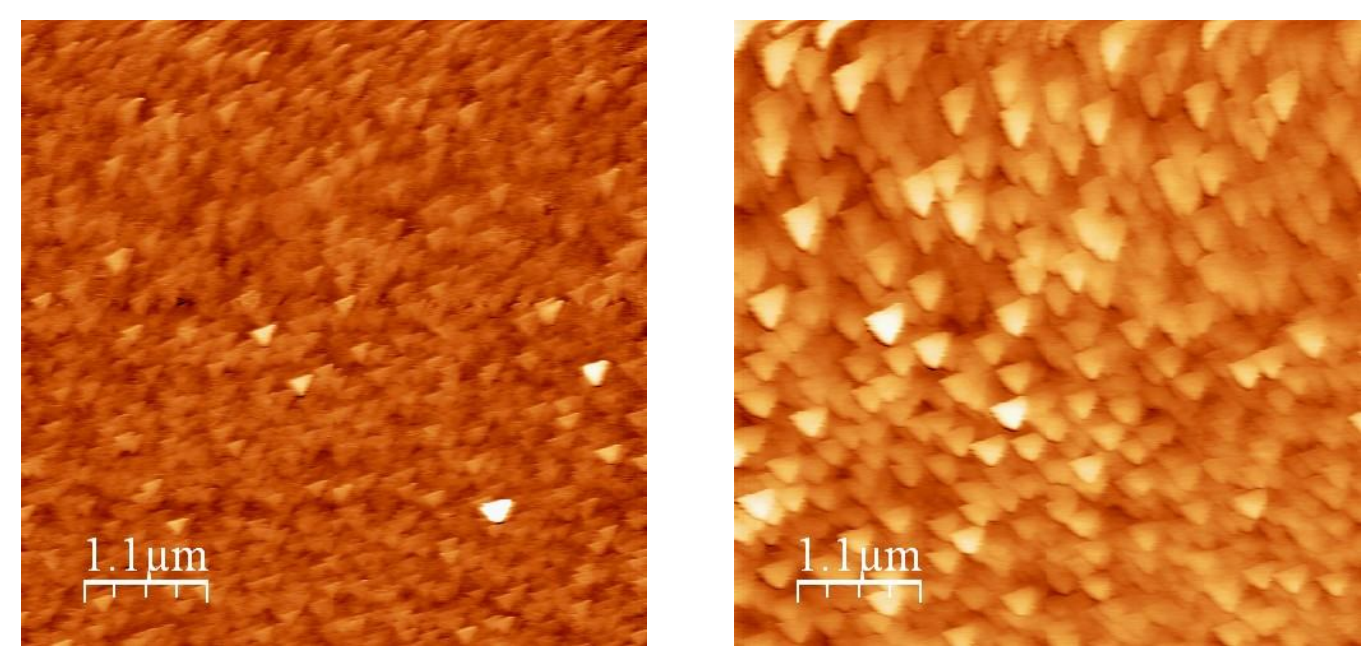

Figure 5. Left: AFM image of $\mathrm{ZnO}$ after being annealed 60 minutes. Right: AFM image of $\mathrm{ZnO}$ after annealed for 90 minutes.

Arbitrary cross sections are commonly used to evaluate the height and width by taking the average of several of these measurements. Before the large change in surface structure, $\mathrm{ZnO}$ exhibits general uniformity of small, fine, triangular grains about $200 \mathrm{~nm}$ wide. After annealing for 90 minutes the triangular grains grew to $350 \mathrm{~nm}$ wide. It is likely these terraces are growing together to make larger ones. Changes in the surface structure could hold important changes in the optical and electrical properties of the film. These terraces are important because their size is related to the resistivity of the surface. As the films were annealed for longer than 90 minutes the grains continued to grow in size at a much slower rate and the uniformity of the films were lost. 

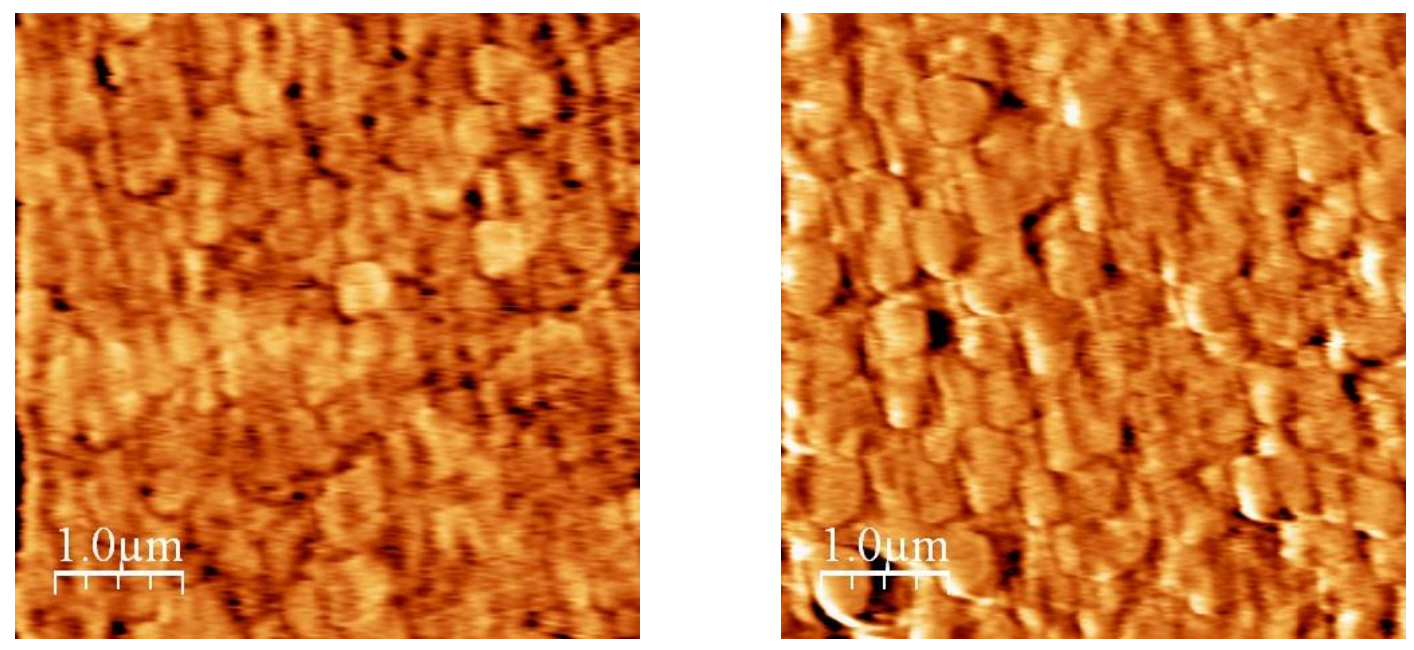

Figure 6. On the left is an AFM image of a $\mathrm{ZnO} / \mathrm{Cu}$ nanolaminate sample after 60 minutes annealed. On the right is an image of the same sample with an additional 30 minutes annealed.

After the pure $\mathrm{ZnO}$ films were analyzed the $\mathrm{ZnO} / \mathrm{Cu}$ nanolaminate films were annealed and evaluated. The largest change in surface structure occurred over the same time interval as the pure $\mathrm{ZnO}$ films. Figure 6 shows the change in the surface from 60 to 90 minutes annealed. As expected, the cross sectional analysis again shows an increase in grain sizes; from $400 \mathrm{~nm}$ to $550 \mathrm{~nm}$ in the 30 minutes shown.

\section{Conclusion}

After sputter deposition the thin $\mathrm{ZnO}$ film has a surface which may be too rough to be applicable in solar devices. This can be overcome by annealing the sample to create a smoother more compatible surface. The grain sizes are important because their size is related to the resistivity of the surface, a property that is obviously crucial when studying the possibility of implementing into photovoltaic devices. Annealing also increases uniformity, which is ideal for use in semiconductors. As a result, annealing the films for 90 minutes was determined to be the proper amount needed for the desired surface changes. This increase in crystal size should lower the resistance of the film and allow the short circuit current density to increase [5].

The reality of an inexpensive, good electrical conductor with a transparent medium shows the possibility of replacing the TCO layer in photovoltaic devices. With the ability to be produced at a low temperature and its' wide and direct band gap, a $\mathrm{ZnO}$ alloy is a prospect worthwhile. Significant visual changes were found in the annealing process of these films. This study will set the ground work in future work to better understand $\mathrm{ZnO}$ applications in photovoltaic devices. 
References

[1] N. Lior, Energy Conv. And Manage. 42, 1769 (2001)

[2] http://gcep.stanford.edu/research/exergycharts.html

[3] J. C. Bernède, Y. Berredjem, L. Cattin, and M. Morsli , Appl. Phys. Lett. 92, 083304 (2008)

[4] Z. Li and W. Gao, Mat. Lett. 58, 1363 (2004)

[5] H.H. Afify, S.H. El-Hefnawi, A.Y. Eliwa, M.M. Abdel-Naby, and N.M. Ahmed, Egypt. J. Solids 28, 243 (2005) 